Article

\title{
Optimal Scanning Protocols for Dual-Energy CT Angiography in Peripheral Arterial Stents: An in Vitro Phantom Study
}

\author{
Abdulrahman Almutairi ${ }^{1,2}$, Zhonghua Sun ${ }^{1, \dagger, *}$, Zakariya Al Safran ${ }^{2, \dagger}$, \\ Abduljaleel Poovathumkadavi ${ }^{2, \dagger}$, Suha Albader ${ }^{2, \dagger}$ and Husam Ifdailat ${ }^{2}$ \\ 1 Department of Medical Radiation Sciences, Curtin University, \\ Perth, 6102 Western Australia, Australia; E-Mail: aa1ss@hotmail.com \\ 2 Department of Medical Imaging, King Fahad Specialist Hospital, Dammam 31444, Saudi Arabia; \\ E-Mails: doctorsafranzak@yahoo.com (Z.A.S.); drjaleelpoovathum@gmail.com (A.P.); \\ s_albadr@yahoo.com (S.A.); hfdielat@gmail.com (H.I.) \\ $\dagger$ These authors contributed equally to this work. \\ * Author to whom correspondence should be addressed; E-Mail: z.sun@curtin.edu.au; \\ Tel.: +61-8-9266-7509; Fax: +61-8-9266-2377.
}

Academic Editor: William Chi-shing Cho

Received: 2 February 2015 / Accepted: 8 May 2015 / Published: 20 May 2015

\begin{abstract}
Objective: To identify the optimal dual-energy computed tomography (DECT) scanning protocol for peripheral arterial stents while achieving a low radiation dose, while still maintaining diagnostic image quality, as determined by an in vitro phantom study. Methods: Dual-energy scans in monochromatic spectral imaging mode were performed on a peripheral arterial phantom with use of three gemstone spectral imaging (GSI) protocols, three pitch values, and four kiloelectron volts $(\mathrm{keV})$ ranges. A total of 15 stents of different sizes, materials, and designs were deployed in the phantom. Image noise, the signal-to-noise ratio (SNR), different levels of adaptive statistical iterative reconstruction (ASIR), and the four levels of monochromatic energy for DECT imaging of peripheral arterial stents were measured and compared to determine the optimal protocols. Results: A total of 36 scans with 180 datasets were reconstructed from a combination of different protocols. There was a significant reduction of image noise with a higher SNR from monochromatic energy images between 65 and $70 \mathrm{keV}$ in all investigated preset GSI protocols $(p<0.05)$. In addition, significant effects were found from the main effect analysis for these factors: GSI, pitch, and $\mathrm{keV}(p=0.001)$. In contrast, there was significant interaction on the
\end{abstract}


unstented area between GSI and ASIR $(p=0.015)$ and a very high significant difference between $\mathrm{keV}$ and ASIR $(p<0.001)$. A radiation dose reduction of $50 \%$ was achieved. Conclusions: The optimal scanning protocol and energy level in the phantom study were GSI-48, pitch value 0.984 , and $65 \mathrm{keV}$, which resulted in lower image noise and a lower radiation dose, but with acceptable diagnostic images.

Keywords: dual-energy CT; gemstone spectral imaging; image noise; monochromatic image; peripheral arterial stent

\section{Introduction}

The increasing prevalence of peripheral artery disease (PAD) is an important cardiovascular disease risk factor [1-3]. Stent placement for occlusive vascular disease is recognized as a safe and effective alternative treatment for PAD [4]. The main concern of stent implantation is the development of in-stent restenosis. Recent studies have shown a 30\%-55\% restenosis rate after the first year of stent implementation [5-7], indicating that a follow-up examination for the patency of implanted stents is important. A number of imaging techniques have been used to evaluate stent patency, including digital subtraction angiography (DSA), multi-detector computed tomography (MDCT), Doppler ultrasound, and magnetic resonance imaging. Doppler ultrasound is a non-invasive technique, which allows measurement of blood flow to confirm the diagnosis of occlusive PAD. However, the use of Doppler ultrasound is restricted when vascular are calcified or stented. Its diagnostic accuracy also depends on both the operator's experience and the patient's body habitus. Magnetic resonance angiography, on the other hand, could represent an alternative, non-invasive approach. However, for stents evaluation this procedure might be limited due to signal decrease or signal loss caused by metallic stents. Although DSA was the standard follow-up procedure for PAD, there are some disadvantages of this modality, which include invasiveness and limited assessment to the vessel structures. Therefore, it has gradually been replaced by less invasive techniques, such as MDCT [8,9], because MDCT is associated with few procedure-related complications, but with shorter procedural time, and fewer motion artifacts [8,9]. Despite these advantages, MDCT has its weaknesses, including a higher rate of contrast medium-induced nephrotoxicity, suffering from blooming artifacts caused by stent struts, and risk of high radiation dose.

The latest MDCT systems, such as dual-source computed tomography (DSCT) and dual-energy computed tomography (DECT), are capable of addressing these weaknesses [10]. In particular, DECT has the ability to distinguish different materials at high density-for example, separating iodinated contrast from other materials [11]. In addition, beam-hardening artifacts, which usually result from the polychromatic energy of the X-ray spectrum, can be eliminated by using the monochromatic energy images (MEI) spectrum. Furthermore, shorter rotation time and use of iterative reconstruction can reduce the radiation dose [12]. Although extensive studies have been conducted on the use of DECT for cardiovascular disease, there is a paucity of literature focusing on the lower extremities, especially for stent patency evaluation [13-16].

Most of the previous studies on DECT were performed on dual-source DECT, which uses two X-ray tubes [8,16-19]. However, the fast kilovoltage switching CT scanner, using one X-ray tube and a full 
field of view (FOV) with special detector gemstone spectral image (GSI) of GE medical systems, represents another advantage of DECT, as it can improve image quality by reducing beam-hardening artifacts associated with stents. Furthermore, it can distinguish between materials such as contrast and soft tissue or other materials by suppressing one material and enhancing the other with better temporal registration [20]. To the best of our knowledge, there is no report available in the literature on using the fast kilovoltage switching GE scanner for evaluation of periperal arterial stents. Thus, the purpose of this study is to identify an optimal DECT scanning protocol that provides a lower radiation dose and maintains image quality in peripheral arterial stents based on an in vitro phantom study.

\section{Results}

A total of 180 series acquired with virtual MEI imaging at 4-kiloelectron volts (keV) and 5-adaptive statistical iterative reconstruction (ASIR) levels were reconstructed to determine the interaction between peripheral arterial stent image quality factors and scanning protocols. There was a significant reduction of image noise with MEI between 65 and $70 \mathrm{keV}$ in all investigated preset GSI protocols $(p<0.05)$. A significant reduction was observed at $65 \mathrm{keV}$ for the unstented area, and for large diameter stents and small diameter stents with both GSI-48 and GSI-51. However, the mean HU was reduced as the $\mathrm{keV}$ increased for all protocols, as shown in Figure 1E. Results indicated that the preset GSI-48 scanning protocol with a pitch value of $0.984,65 \mathrm{keV}$, and ASIR $\leq 50 \%$ achieved the optimal image quality compared with the other protocols, as shown in Figure 1A-E. Figure 2 shows an example of a series of images acquired with protocols using $65 \mathrm{keV}$ and 3 GSI settings.



(A)


(B)

Figure 1. Cont. 



(C)


(D)


(E)

Figure 1. (A-C) show the comparison of Noise Level at different kiloelectron voltage $(\mathrm{keV})$ with the three preset GSI protocols and three pitch values, while Figure $(\mathbf{D}, \mathbf{E})$ represent the mean of $\mathrm{CT}$ value in unstented area and all stents with these scanning protocols. 



(A)


GSI-48 Pitch 1.375at $65 \mathrm{keV}$

(B)

Figure 2. Cont. 



(C)

Figure 2. A total of 13 stents (No. 7 and 14 were not included due to difficulty placing region of interest in the area) with axial and coronal reformatted images were demonstrated with three GSI protocols (A-C: GSI-36, GSI-48, and GSI-51, respectively) and three pitch values at a $\mathrm{keV}$ of 65 .

\subsection{Image Quality Assessment}

The phantom data were analyzed with a 3-GSI * 3-Pitch * 4-keV factorial ANOVA. Each effect was tested with a mean standard error (MSE) of 71.27. There was highly significant interaction of image noise and SNR with the GSI and pitch $(p=0.001)$. Similar findings were observed in the unstented area with highly significant effects. In addition, significant effects were found for these factors-GSI, pitch, and $\mathrm{keV}(p=0.001)$. In contrast, there was another significant interaction on the unstented area between GSI and ASIR $(p=0.015)$ and a very highly significant difference between $\mathrm{keV}$ and ASIR $(p<0.001)$. Although for noise level, two of the 3-way interactive terms were statistically significant, namely the GSI, pitch, and keV, and the GSI, pitch, and ASIR $(p<0.001)$; the main effects and interactions for these are shown in Table 1. The noise level (MSE = 89.04) was higher in GSI-36 than in the other two GSIs, as shown in Figure 3A-E. 
Table 1. Results of factorial ANOVA.

\begin{tabular}{|c|c|c|c|c|c|c|c|c|c|}
\hline \multirow{2}{*}{\multicolumn{2}{|c|}{ Effects }} & \multicolumn{2}{|c|}{ SNR } & \multicolumn{2}{|c|}{ SNR2 } & \multicolumn{2}{|c|}{ NL } & \multicolumn{2}{|c|}{ NL2 } \\
\hline & & \multirow{2}{*}{$\begin{array}{c}\boldsymbol{F} \\
11.806\end{array}$} & \multirow{2}{*}{\begin{tabular}{|c|}
$\boldsymbol{P}$ \\
0.001
\end{tabular}} & \multirow{2}{*}{$\begin{array}{c}\boldsymbol{F} \\
86.658\end{array}$} & \multirow{2}{*}{\begin{tabular}{|c|}
$\boldsymbol{P}$ \\
0.000
\end{tabular}} & \multirow{2}{*}{$\begin{array}{c}\boldsymbol{F} \\
947.509\end{array}$} & \multirow{2}{*}{\begin{tabular}{|c|}
$\boldsymbol{P}$ \\
0.000
\end{tabular}} & \multirow{2}{*}{$\begin{array}{c}\boldsymbol{F} \\
572.661\end{array}$} & \multirow{2}{*}{$\begin{array}{c}\boldsymbol{P} \\
0.000\end{array}$} \\
\hline GSI & Main effect & & & & & & & & \\
\hline Pitch & Main effect & 10.992 & 0.002 & 302.411 & 0.000 & 102.664 & 0.000 & 1883.182 & 0.000 \\
\hline $\mathrm{KeV}$ & Main effect & 13.424 & 0.000 & 192.161 & 0.000 & 230.042 & 0.000 & 894.644 & 0.000 \\
\hline ASIR & Main effect & NA & NA & 157.416 & 0.000 & NA & NA & 1291.264 & 0.000 \\
\hline GSI * ASIR & Two-factor interaction effect & NA & NA & 3.823 & 0.015 & NA & NA & 27.164 & 0.000 \\
\hline $\mathrm{KeV} * \mathrm{ASIR}$ & Two-factor interaction effect & NA & NA & 6.133 & 0.001 & NA & NA & 3.652 & 0.010 \\
\hline Pitch * ASIR & Two-factor interaction effect & NA & NA & 1.877 & 0.147 & NA & NA & 68.25 & 0.000 \\
\hline $\mathrm{GSI} * \mathrm{KeV}$ & Two-factor interaction effect & 0.454 & 0.829 & 16.528 & 0.000 & 0.649 & 0.691 & 120.311 & 0.000 \\
\hline GSI * Pitch & Two-factor interaction effect & 13.708 & 0.000 & 12.793 & 0.000 & 142.367 & 0.000 & 30.586 & 0.000 \\
\hline Pitch $* \mathrm{KeV}$ & Two-factor interaction effect & 0.419 & 0.853 & 18.778 & 0.000 & 1.189 & 0.375 & 120.391 & 0.000 \\
\hline GSI $* \mathrm{KeV} *$ ASIR & Three-factor interaction effect & NA & NA & 0.457 & 0.920 & NA & NA & 1.727 & 0.123 \\
\hline GSI * Pitch * ASIR & Three-factor interaction effect & NA & NA & 0.634 & 0.742 & NA & NA & 9.842 & 0.000 \\
\hline Pitch $* \mathrm{KeV} *$ ASIR & Three-factor interaction effect & NA & NA & 0.558 & 0.853 & NA & NA & 1.326 & 0.268 \\
\hline GSI $*$ Pitch $* \mathrm{KeV}$ & Three-factor interaction effect & NA & NA & 21.545 & 0.000 & NA & $\mathrm{NA}$ & 118.371 & 0.000 \\
\hline
\end{tabular}

SNR: Signal to noise ratio in the stented area; SNR2: Signal to noise ratio in the unstented area; NL: noise level in the stented area; NL2: noise level in the unstented area; $F$ : value of test statistic of $F$-test for corresponding effect; $P$ : corresponding $p$ value; GSI: gemstone spectral image protocol; keV: kiloelectron volt; ASIR: adaptive statistical iterative reconstruction; NA: not applicable; * = multiplication. 




(A)



(C)

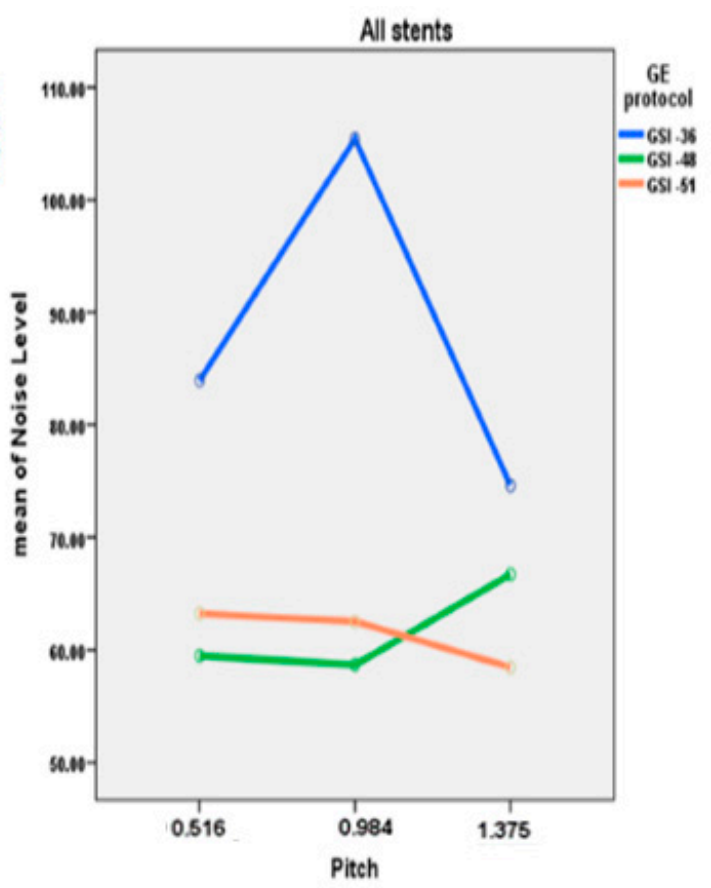

(B)

Medium Stents



(D)

Figure 3. Cont. 




(E)



(F)

Figure 3. (A-E) A comparison of relationship between noise levels measured with different GSI protocols and pitch values at $65 \mathrm{keV}$ with different diameters of stents; (F) represents the mean of CT values measured in all stents with use of three GSI and pitch protocols.

\subsection{Effect of keV on Image Quality}

There was no significant interaction between $\mathrm{keV}$ and GSI or pitch factors in the stented area. However, this does not mean that there was no effect of $\mathrm{keV}$ on image quality, but the effect of $\mathrm{keV}$ on image noise was not dependent on GSI or pitch. Therefore, the four noise-level means for $\mathrm{keV}$ ranged from 83.94 to $64.30 \mathrm{HU}$ and, incidentally, decreasing image noise occurred monotonically with increasing $\mathrm{keV}$. Thus, the effect of $\mathrm{keV}$ on image noise was independent of any other design effect. A similar effect was observed with the SNR. Unlike its effect on the unstented area, the effect of $\mathrm{keV}$ was found to be highly significant for both image noise and SNR $(p=0.001)$. Figure 4 compares the selected $\mathrm{keV}$ in different GSIs for both unstented and stented areas with different ASIR values.



(A)



(B)

Figure 4. Cont. 




(C)

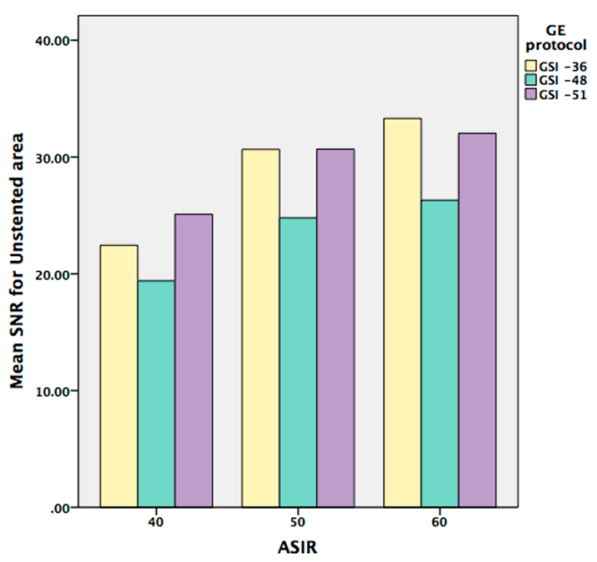

(D)

Figure 4. $(\mathbf{A}, \mathbf{B})$ are graphic representations showing the noise level when ASIR is used within the both unstented and stented areas; while $(\mathbf{C}, \mathbf{D})$ represent the means of SNR when ASIR is used.

\subsection{Effect of GSI and Pitch on Image Quality}

The interaction of GSI by pitch is highly statistically significant $(p<0.001)$. A significance interaction was observed on the unstented area. Also, the main effects of GSI and pitch on noise level are highly significant $(p<0.001)$, as shown in Table 1 .

The marginal noise level means for the 3-GSI categories were 89.03, 62.86, and 61.92, indicating significant differences with the first GSI and with both the second and third GSI of around 27.11 and $0.94 \mathrm{HU}$, respectively. However, when the three GSI means were investigated for the first pitch category $(84.90,60.60$, and 63.95), significant changes were observed. These changes varied both with respect to magnitude and direction, and presented as the significant interaction of GSI by pitch.

\subsection{Effect of Type of Stents on Image Quality}

The noise level in the unstented area showed a direct relationship between image noise and pitch value, with noise increasing when pitch increased. However, with large stents, lower image noise was found in GSI-36 and GSI-48 protocols, with a pitch value of 0.984. The medium sized stent showed that a lower noise was achieved by a GSI-48 and pitch of 0.984 , while result is different from that for the small stents where the best visualization was achieved with use of GSI-51 and pitch of 0.516 , as shown in Figure 3.

A radiation dose reduction of about $50 \%$ was achieved in all protocols when the pitch value 0.984 was used. However, a minimal reduction was observed when the pitch value was changed from 0.984 to 1.375 .

\subsection{Subjective Image Quality Assessment}

The subjective grading of image quality showed a discrepancy between the readers with a kappa value of 0.24 . This might be explained by the limited experience of those readers in clinical research. The box plots of the noise level for the three radiologists are shown in Figure 5. 




Figure 5. Box plots demonstrate the radiologist's evaluation of the image quality using a 3 -point scale. The red line within the yellow box indicates the median of the values. The red line at the bottom of the box corresponds to the 25 th percentile, and the one at the top is the 75th percentile. The range between these two lines (i.e., the length of the box) is the inter Quartile Range (IQR). The lower cross line is $1.5 \mathrm{IQR}$ below the 25th percentile, the upper cross line is $1.5 \mathrm{IQR}$ above the 75 th percentile. Individual observations beyond either of these cross line are outliners, shown as green circles.

\section{Discussion}

This phantom study indicates that the effect of the pitch factor on the $\mathrm{keV}$ and radiation dose is an important indicator for determining both radiation dose and image quality, as the lowest pitch is associated with the highest radiation dose and vice versa. The lowest radiation dose with sufficient image noise was obtained with the GSI-48 protocol with a pitch of 0.984 and $65 \mathrm{keV}$. Overall, the GSI-51 protocol achieved the highest SNR and lowest noise level values with pitch values of 0.516 and 1.375 ; however, a pitch value of 0.516 was associated with the highest radiation dose, whereas a pitch value of 1.375 was associated with the highest image noise. To our knowledge, no studies have been published comparing different DECT protocols with different pitch values. Results of this study show that the effect of the pitch value on image noise was strongly dependent on rotation time, as the protocol with the shorter rotation time (GSI-36) achieved both the highest SNR and the lowest image noise, and the protocol with the longer rotation time (GSI-51) achieved the highest image noise, even when mAs was higher than that of the other protocols.

It was found that the images acquired with approximately $65-70 \mathrm{keV}$ had less image noise and higher SNR compared to other energies that have a lower noise level and lower SNR. Our results are similar to those reported from a chest study by Cheng et al [15] who found that the MEI images at 65-70 keV resulted in less image noise and a better contrast-to-noise ratio. The findings of the current 
study are consistent with those of $\mathrm{Yu}$ et al [21], who studied various phantom sizes to evaluate MEI at multiple keV levels to optimize chest image quality. They found that the best image quality was obtained with energies of $66 \mathrm{keV}$ for small phantoms, $68 \mathrm{keV}$ for medium, $70 \mathrm{keV}$ for large, and $72 \mathrm{keV}$ for extra-large phantoms [21]. Similarly, Matsumoto et al. [22] reported that using $70 \mathrm{keV}$ achieved the lowest image noise based on a phantom study. Furthermore, Pehno et al [13] compared the subjective and objective image quality of virtual MEI DECT angiography (DECTA) to conventional polyenergetic images (PEI) in aortoillic arteries, demonstrating optimal contrast enhancement and improved image quality using $70 \mathrm{keV}$ MEI compared to single-energy CTA. However, our findings show that the highest image noise was found with $60 \mathrm{keV}$, which differs from those reported by Sudarski et al. [23]. They found that the use of $60 \mathrm{keV}$ for lower extremities led to the best image quality when compared to the quality of PEIs. These findings can be justified because the effect of stents on image noise is clearly evident when comparing small, medium, and large stents with different keV, as shown in Figure 5. The findings from these studies suggest that using keV between 65 and 70 with a pitch value of 0.984 achieves optimal image quality with a lower radiation dose in peripheral arterial DECT.

When iterative reconstruction is evaluated there is a significant difference between the unstented and stented areas. In the unstented area, the image quality was improved when the ASIR was increased from $40 \%$ to $50 \%$. This is similar to previous studies that showed that an ASIR of less than $40 \%$ did not improve the image quality when compared with conventional PEIs [14,24-26]. However, the current study shows that images with stents were not affected by any level of ASIR when they were applied with all of the preset GSI protocols. Therefore, based on the unstented area results, we recommend the use of $50 \% \mathrm{ASIR}$, with 65 to $70 \mathrm{keV}$ in the peripheral arterial stent protocol, and preset GSI-48 as the optimal protocol to replace the conventional CTA.

The optimal protocol that has been evaluated and identified in this study is currently being tested in a clinical study to validate its clinical value and outcome. Despite these protocols being tested on a GE scanner, results of this study can be applicable to other MDCT manufacturers as long as the dual-energy function is available on these scanners.

Our study has some limitations. First, although the experimental setup was developed to simulate a peripheral vascular tree, the idealized anatomic environment did not have surrounding organs, vessel walls, or tissues. The nature of body vessels varies from those in the phantom, and this could affect visualization of stents to some extent. Another limitation is that this custom-made phantom represented only an average-sized adult, while absorption of low-energy radiation will differ for large- or small-sized patients. Finally, the default manufacturer's setting of 0.3 as the weighting factor for the low-energy tube was used to create virtual $120 \mathrm{KV}$ reconstructions; however, there have been reports that a weighting factor of 0.5 improves image quality and therefore would be better for vascular imaging [27]. This suggests that further studies are necessary to confirm our findings.

\section{Experimental Section}

\subsection{Peripheral Artery Phantom Design and Stent Placement}

The custom-made peripheral arterial phantom consisting of a main peripheral arterial tree and arterial branches was developed with use of a computer-aided design program to represent realistic 
anatomic dimensions. The phantom was made of poly methyl methacrylate material with anatomical dimensions similar to the normal anatomy of a peripheral arterial tree, as shown in Figure 6.

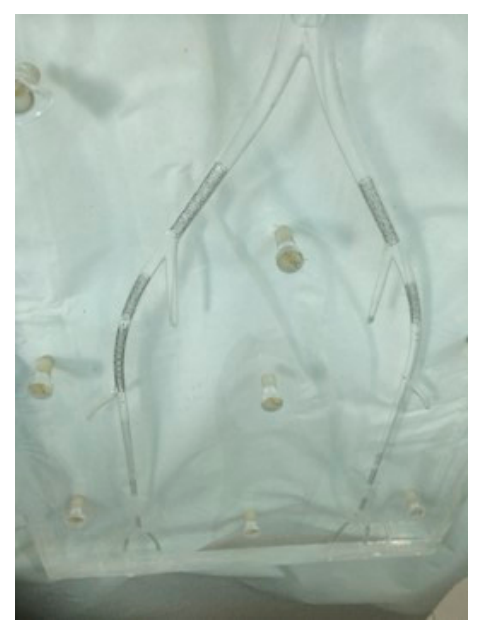

Figure 6. Photograph of the phantom with stents insertion.

A total of 15 expired stents with different sizes (diameters), materials, and designs were used in the experiments. Details of the stents are shown in Table 2. Of these fifteen stents, 10 were made of stainless steel (316 L), 3 of a platinum chromium alloy, 1 of Nitinol, and 1 of cobalt-superalloy. Stents were deployed into the simulated peripheral arteries modeled within the phantom. Stents with lumen diameters ranging from 2.75 to $3,4,5,6,7$, and $8 \mathrm{~mm}$, which closely matched their nominal diameter, were inserted into the arteries with two exceptions: the two Taxus Element stents with nominal diameters of $3 \mathrm{~mm}$ were inserted into simulated arteries with a diameter of $2.5 \mathrm{~mm}$.

\subsection{DECT Scanning Protocols and Image Reconstruction}

DECT scans were performed with a fast kilovoltage-switching 64-slice CT scanner (Discovery CT750 HD; GE Healthcare, Milwaukee, WI, USA). Of the GSI-protocols that the manufacturer has developed as default settings, 3 GSI protocols (GSI preset protocols 36, 48, and 51) were selected for this study based on the lowest $\mathrm{CTDI}_{\mathrm{vol}}$ with acceptable diagnostic image quality to evaluate peripheral arterial stents. These GSI preset protocols have been fixed by the manufacturer to maintain constant tube power when switching the tube voltage back and forth. Therefore, changing the radiation dose in these preset protocols was only possible by adjusting the pitch and/or rotation time. As a result, the three available pitch values, 0.516 , 0.984 , and 1.375 were tested, with a beam collimation of $40 \mathrm{~mm}$ in all of these protocols and fixed rotation times of $0.5,0.7$, and 0.8 second per rotation, respectively, for each protocol. The phantom was then positioned in the gantry in an orientation parallel to the $z$-axis of the scanner.

The scanning parameters for the selected preset GIS-protocols are summarized in Table 3. Images were acquired with coverage of $30 \mathrm{~cm}$ ranging from aortic bifurcation to arteries below knee. The raw data obtained from each scan were reconstructed in four image sets with five levels of adaptive statistical iterative reconstruction (ASIR), $0 \%, 30 \%, 40 \%, 50 \%$, and $60 \%$, and four $\mathrm{keV}$ of 60,65 , 70, and 75, respectively, with slice thicknesses of $1 \mathrm{~mm}$ with $50 \%$ reconstruction overlap. Tube current modulation was not available in dual energy acquisition in this system for all protocols. 
Table 2. Details of the examined stents.

\begin{tabular}{|c|c|c|c|c|c|c|}
\hline Model & Material & Manufacturer & Diameter (mm) & Length $(\mathrm{mm})$ & Stent Diameter & Stents No. \\
\hline Express LD & 316 L stainless steel & Boston Scientific & $7-8$ & $27-37$ & Large & $(1,2,9$ and 10$)$ \\
\hline Absolute .035 & Nitinol & Abbott & 6 & 40 & Medium & 3 \\
\hline Wallstent-Uni Endoprosthesis & Cobalt-superalloy & Boston Scientific & 5 & 40 & Medium & 4 \\
\hline Palmaz Genesis & 316 L Stainless steel & Cordis & 5 & 14 & Medium & $(5,6$ and 11$)$ \\
\hline Taxus Element & 316 L Stainless steel & Boston Scientific & 2.75 & 32 & Small & $(7,14)$ \\
\hline Taxus Libert 2nd Generation & Platinum Chromium & Boston Scientific & 3 & 28 & Small & 8 \\
\hline Promus Element & Platinum Chromium & Boston Scientific & 4 & 16 & Medium & 12 \\
\hline Express Vascular SD & 316 L Stainless steel & Boston Scientific & 4 & 15 & Medium & 13 \\
\hline Monorail Liberté & 316 L Stainless steel & Boston Scientific & 3 & 28 & Small & 15 \\
\hline
\end{tabular}

Table 3. Details of scan parameters by protocols.

\begin{tabular}{cccc}
\hline Scan Parameters & Protocol 1 & Protocol 2 & Protocol 3 \\
\hline GSI protocol & GSI-36 & GSI-48 & GSI-51 \\
Scan mode & Dual-energy & Dual-energy & Dual-energy \\
Tube potential & $80 / 140 \mathrm{kV}$ & $80 / 140 \mathrm{kV}$ & $80 / 140 \mathrm{kV}$ \\
Tube current & $260 \mathrm{mAs}$ & $260 \mathrm{mAs}$ & $360 \mathrm{mAs}$ \\
Rotation time (s) & 0.8 & 0.7 & 0.5 \\
Detector collimation (mm) & $64 \times 0.625$ & $64 \times 0.625$ & $64 \times 0.625$ \\
Pitch & $0.516,0.984$ and 1.375 & $0.516,0.984$ and 1.375 & $0.516,0.984$ and 1.375 \\
Table speed (mm/R) & 20.62 & 39.37 & 55 \\
Reconstruction kernel & & Standard & 1 \\
Section thickness (mm) & 1 & 1 & 0.5 \\
Interval & 0.5 & 0.5 & \\
keV & & $(60,65,70$, and 75$)$ & \\
ASIR & & $(30,40,50$, and 60$)$ & \\
\hline
\end{tabular}


A simulated intravenous contrast medium (Omnipaque 350, GE Healthcare, Milwaukee, WI, USA) was used to represent the actual contrast-enhanced CT angiography. The contrast medium was diluted with normal saline to reach the attenuation of 250 Hounsfield unit (HU), which is the acceptable CT attenuation in peripheral CT angiography. The contrast medium was injected into the simulated arteries, which were sealed at both ends [28].

\subsection{Quantitative Image Assessment}

Quantitative measurements were performed for DECT images at $1 \mathrm{~mm}$ slice thickness on a separate independent workstation with the GSI Viewer (ADW 4.6 General Electric Healthcare, Milwaukee, WI, USA). The mean HU was obtained by placing a circular region of interest (ROI) in selected areas of the phantom for the 15 stents (iliac arteries, common femoral arteries, superficial femoral arteries, popliteal arteries, anterior tibial arteries, peroneal arteries, and posterior tibial arteries). Two ROIs were selected; the first one was placed in the common femoral artery to measure the noise on an unstented area of the phantom. The second ROI was placed in the stented lumen area on the axial images to measure the noise for all stents without inclusion of stent struts, as shown in Figure 7. For two stents-No. 7 and No. 14-it was difficult to place the ROI due to the small size of the stents with a limited lumen area being visualized; therefore, we excluded them from the analysis.

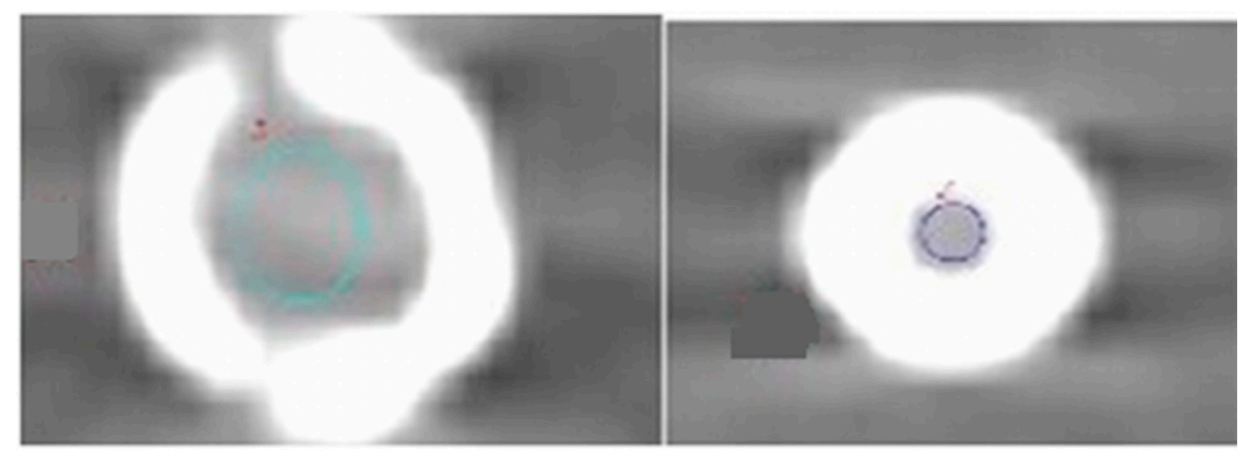

Figure 7. The region of interest was placed in the inner stent lumen for measurement of image noise. The colored circles indicate measurement inside the stent lumen.

The selected ROI in an unstented area was in the femoral artery and within each stent at the same location for all protocols to measure the mean CT attenuation and also image noise that was defined as the standard deviation (SD). Moreover, the signal-to-noise ratio (SNR) for both the stented and unstented areas was calculated with the following formula:

$$
\mathrm{SNR}=\text { in stent lumen or nonstented area of CT attenuation in HU/SD }
$$

\subsection{Qualitative Assessment of Image Quality}

Three radiologists with 5, 15, and 20 years of experience in CT imaging, respectively, performed qualitative evaluation on the GSI viewer with identical window width, window level, and FOV. Different MEI series from the each GSI protocol were evaluated randomly (between 60, 65, 70 and $75 \mathrm{keV}$ within each ASIR), with a total of 180 series reviewed by each reviewer. The readers were 
blinded to all scanning parameters, except for the different energies, because different values of $\mathrm{keV}$ could easily be determined by visual inspection of the images. Intraobserver variability was not estimated because each radiologist assessed the images only once. Visualization of the stent lumen was assessed using a 3-point scale $(1=$ poor image quality and non-diagnostic; 2 = adequate image quality; and $3=$ good image quality).

\subsection{CT Dose}

A fixed scan length of $29.9 \mathrm{~cm}$ was used for all examinations. The volume CT dose index (CTDIvol) and dose-length products (DLP) were recorded for calculation of effective dose, as shown in Table 4. Effective dose (ED) was calculated for each protocol using a conversion factor of 0.015 , which is taken from the normalized value of the effective dose per dose-length product for peripheral arteries [29].

Table 4. Summary of CTDI $\mathrm{vol}_{\mathrm{v}}$ values, dose-length products, and effective doses across the protocols.

\begin{tabular}{ccccc}
\hline GE Protocol & Pitch & CTDI $_{\mathbf{v o l}}(\mathbf{m G y})$ & DLP $(\mathbf{m G y} \times \mathbf{c m})$ & Effective Dose $(\mathbf{m S v})$ \\
\hline GSI-36 & 0.516 & 39.33 & 863.86 & 12.96 \\
GSI-36 & 0.984 & 10.30 & 457.56 & 6.86 \\
GSI-36 & 1.375 & 7.73 & 341.00 & 6.47 \\
GSI-48 & 0.516 & 17.28 & 759.13 & 11.39 \\
GSI-48 & 0.984 & 9.05 & 402.10 & 6.03 \\
GSI-48 & 1.375 & 6.48 & 299.72 & 4.50 \\
GSI-51 & 0.516 & 19.74 & 867.57 & 13.01 \\
GSI-51 & 0.984 & 10.34 & 459.66 & 6.89 \\
GSI-51 & 1.375 & 7.40 & 342.69 & 5.14 \\
\hline
\end{tabular}

GSI, gemstone spectral imaging; $\mathrm{CTDI}_{\mathrm{vol}}$, computed tomography volume dose index; DLP, dose-length product.

\subsection{Statistical Analysis}

All of the data were entered into SPSS Statistics version 22.0 (SPSS Inc., Chicago, IL, USA) for statistical analysis. Two data sets - the noise level and SNR (independent variable) responses obtained when ASIR $=0$ - were used to produce 36 observations in a 3-factor factorial analysis of variance (univariate ANOVA) model: GSI (3-levels: GSI-36, GSI-48, GSI-51), pitch (3-levels: 0.516, 0.984, 1.375), and keV (4-level: 60, 65, 70, 75).

The second part of the statistical analysis relates to the noise level and SNR in the nonstented area when ASIR $\geq 40$; 3-levels: 40, 50, 60 were used to produce 108 observations in a 4-factor factorial model. The highest order interaction in each analysis was employed as an estimate of residual (error) variation; therefore, when there was no significant interaction, the main effect was considered. Statistical significance was assessed by comparing the $p$ values obtained in the $F$-tests corresponding to the main effect of GSI as well as to the interaction effect of pitch, $\mathrm{keV}$, and ASIR. All tests were performed at the $5 \%$ significance level. 


\section{Conclusions}

In conclusion, all preset GSI protocols were found to be suitable for the evaluation of peripheral arterial stents. This study recommends use of the faster rotation time with a pitch value of 0.984 and $\mathrm{keV}$ of $65-70$ with $50 \%$ ASIR for peripheral arterial stent visualization with DECT, as this protocol results in both lower image noise and radiation dose, but with acceptable diagnostic images.

\section{Acknowledgments}

We are grateful to Gil Stevenson for his assistance in the data analysis.

\section{Author Contributions}

Conceived and designed the experiments: Aldulrahman Almutairi, Zhonghua Sun. Performed research: Abdulrahman Almutairi. Wrote the paper: Aldulrahman Almutairi, Zhonghua Sun. Data analysis and Assessment: Aldulrahman Almutairi, Zhonghua Sun, Zakariya Al Safran, Abduljaleel Poovathumkadavi, Suha Albader, Husam Ifdailat. Contribution to the critical appraisal of the paper and approved the final version: Aldulrahman Almutairi, Zhonghua Sun.

\section{Conflicts of Interest}

The authors declare no conflict of interest.

\section{References}

1. Rosamond, W.; Flegal, K.; Furie, K.; Go, A.; Greenlund, K.; Haase, N.; Hailpern, S.M.; Ho, M.; Howard, V.; Kissela, B.; et al. Heart disease and stroke statistics-2008 update: A report from the American Heart Association Statistics Committee and Stroke Statistics Subcommittee. Circulation 2008, 117, e25-e146.

2. Norgren, L.; Hiatt, W.R.; Dormandy, J.A.; Nehler, M.R.; Harris, K.A.; Fowkes, F.G.R.; Bell, K.; Caporusso, J.; Durand-Zaleski, I.; Komori, K.; et al. In inter-society consensus for the management of peripheral arterial disease (TASC II). Eur. J. Vasc. Endovasc. Surg. 2007, 33, S1-S75.

3. Fowkes, F.G.R.; Rudan, D.; Rudan, I.; Aboyans, V.; Denenberg, J.O.; McDermott, M.M.; Norman, P.E.; Sampson, U.K.A.; Williams, L.J.; Mensah, G.A.; et al. Comparison of global estimates of prevalence and risk factors for peripheral artery disease in 2000 and 2010: A systematic review and analysis. Lancet 2013, 382, 1329-1340.

4. Napoli, A.; Anzidei, M.; Zaccagna, F.; Cavallo Marincola, B.; Zini, C.; Brachetti, G.; Cartocci, G.; Fanelli, F.; Catalano, C.; Passariello, R. Peripheral arterial occlusive disease: Diagnostic performance and Effect on therapeutic management of 64-Section CT Angiography. Radiology 2011, 261, 976-986.

5. Rastan, A.; Krankenberg, H.; Baumgartner, I.; Blessing, E.; Müller-Hülsbeck, S.; Pilger, E.; Scheinert, D.; Lammer, J.; Gißler, M.; Noory, E.; et al. Stent Placement versus balloon angioplasty for the treatment of obstructive lesions of the popliteal artery: A prospective, multicenter, randomized trial. Circulation 2013, 127, 2535-2541. 
6. Iida, O.; Yokoi, H.; Soga, Y.; Inoue, N.; Suzuki, K.; Yokoi, Y.; Kawasaki, D.; Zen, K.; Urasawa, K.; Shintani, Y.; et al. Cilostazol reduces angiographic restenosis after endovascular Therapy for femoropopliteal lesions in the sufficient treatment of peripheral intervention by cilostazol study. Circulation 2013, 127, 2307-2315.

7. Tendera, M.; Aboyans, V.; Bartelink, M.-L.; Baumgartner, I.; Clément, D.; Collet, J.-P.; Cremonesi, A.; de Carlo, M.; Erbel, R.; Fowkes, F.G.R.; et al. ESC Guidelines on the diagnosis and treatment of peripheral artery diseases. Eur. Heart J. 2011, 32, 2851-2906.

8. Brockmann, C.; Jochum, S.; Sadick, M.; Huck, K.; Ziegler, P.; Fink, C.; Schoenberg, S.O.; Diehl, S.J. Dual-energy CT angiography in peripheral arterial occlusive disease. Cardiovasc. Interv. Radiol. 2009, 32, 630-637.

9. Sun, Z. Diagnostic accuracy of multislice CT angiography in peripheral arterial disease. J. Vasc. Interv. Radiol. 2006, 17, 1915-1921.

10. Coursey, C.A.; Nelson, R.C.; Boll, D.T.; Paulson, E.K.; Ho, L.M.; Neville, A.M.; Marin, D.; Gupta, R.T.; Schindera, S.T. Dual-energy multidetector CT: How does it work, what can it tell us, and when can we use it in abdominopelvic imaging? Radiographics 2010, 30, 1037-1055.

11. Graser, A.; Johnson, T.C.; Chandarana, H.; Macari, M. Dual energy CT: Preliminary observations and potential clinical applications in the abdomen. Eur. Radiol. 2009, 19, 13-23.

12. Silva, A.C.; Morse, B.G.; Hara, A.K.; Paden, R.G.; Hongo, N.; Pavlicek, W. Dual-energy (Spectral) CT: Applications in abdominal imaging. Radiographics 2011, 31, 1031-1046.

13. Pinho, D.F.; Kulkarni, N.M.; Krishnaraj, A.; Kalva, S.P.; Sahani, D.V. Initial experience with single-source dual-energy CT abdominal angiography and comparison with single-energy CT angiography: Image quality, enhancement, diagnosis and radiation dose. Eur. Radiol. 2013, 23, 351-359.

14. Fuchs, T.A.; Stehli, J.; Fiechter, M.; Dougoud, S.; Gebhard, C.; Ghadri, J.R.; Husmann, L.; Gaemperli, O.; Kaufmann, P.A. First experience with monochromatic coronary computed tomography angiography from a 64-slice CT scanner with Gemstone Spectral Imaging (GSI). J. Cardiovasc. Comput. Tomogr. 2013, 7, 25-31.

15. Cheng, J.; Yin, Y.; Wu, H.; Zhang, Q.; Hua, J.; Hua, X.; Xu, J. Optimal monochromatic energy levels in spectral CT pulmonary angiography for the evaluation of pulmonary embolism. PLoS ONE 2013, 8, e63140.

16. Huang, S.Y.; Nelson, R.C.; Miller, M.J.; Kim, C.Y.; Lawson, J.H.; Husarik, D.B.; Boll, D.T. Assessment of vascular contrast and depiction of stenoses in abdominopelvic and lower extremity vasculature: Comparison of dual-energy MDCT with digital subtraction angiography. Acad. Radiol. 2012, 19, 1149-1157.

17. Maaß, C.; Baer, M.; Kachelrieß, M. Image-based dual energy CT using optimized precorrection functions: A practical new approach of material decomposition in image domain. Med. Phys. 2009, 36, 3818-3829.

18. Kau, T.; Eicher, W.; Reiterer, C.; Niedermayer, M.; Rabitsch, E.; Senft, B.; Hausegger, K. Dual-energy CT angiography in peripheral arterial occlusive disease-accuracy of maximum intensity projections in clinical routine and subgroup analysis. Eur. Radiol. 2011, 21, 1677-1686. 
19. Yamamoto, S.; McWilliams, J.; Arellano, C.; Marfori, W.; Cheng, W.; McNamara, T.; Quinones-Baldrich, W.J.; Ruehm, S.G. Dual-energy CT angiography of pelvic and lower extremity arteries: Dual-energy bone subtraction versus manual bone subtraction. Clin. Radiol. 2009, 64, 1088-1096.

20. Krasnicki, T.; Podgorski, P.; Guzinski, M.; Czarnecka, A.; Tupikowski, K.; Garcarek, J.; Marek Sasiadek, M. Novel clinical applications of dual energy computed tomography. Adv. Clin. Exp. Med. 2012, 21, 831-841.

21. Yu, L.; Christner, J.A.; Leng, S.; Wang, J.; Fletcher, J.G.; McCollough, C.H. Virtual monochromatic imaging in dual-source dual-energy CT: Radiation dose and image quality. Med. Phys. 2011, 38, 6371-6379.

22. Matsumoto, K.; Jinzaki, M.; Tanami, Y.; Ueno, A.; Yamada, M.; Kuribayashi, S. Virtual monochromatic spectral imaging with fast kilovoltage switching: Improved image quality as compared with that obtained with conventional 120-kVp CT. Radiology 2011, 259, 257-262.

23. Sudarski, S.; Apfaltrer, P.; Nance, J.J.; Schneider, D.; Meyer, M.; Schoenberg, S.O.; Fink, C.; Henzler, T. Optimization of keV-settings in abdominal and lower extremity dual-source dual-energy CT angiography determined with virtual monoenergetic imaging. Eur. J. Radiol. 2013, 82, e574-e581.

24. Marin, D.; Choudhury, K.; Gupta, R.; Ho, L.; Allen, B.; Schindera, S.; Colsher, J.; Samei, E.; Nelson, R. Clinical impact of an adaptive statistical iterative reconstruction algorithm for detection of hypervascular liver tumours using a low tube voltage, high tube current MDCT technique. Eur. Radiol. 2013, 23, 3325-3335.

25. Kulkarni, N.M.; Uppot, R.N.; Eisner, B.H.; Sahani, D.V. Radiation dose reduction at multidetector CT with adaptive statistical iterative reconstruction for evaluation of urolithiasis: How low can we go? Radiology 2012, 265, 158-166.

26. Vardhanabhuti, V.; Olubaniyi, B.; Loader, R.; Riordan, R.D.; Williams, M.P.; Roobottom, C.A. Image quality assessment in torso phantom comparing effects of varying automatic current modulation with filtered back projection, adaptive statistical, and model-based iterative reconstruction techniques in CT. J. Med. Imaging Radiat. Sci. 2012, 43, 228-238.

27. Behrendt, F.F.; Schmidt, B.; Plumhans, C.; Keil, S.; Woodruff, S.G.; Ackermann, D.; Muhlenbruch, G.; Flohr, T.; Gunther, R.W.; Mahnken, A.H. Image fusion in dual energy computed tomography: Effect on contrast enhancement, signal-to-noise ratio and image quality in computed tomography angiography. Investig. Radiol. 2009, 44, 1-6.

28. Kohler, M.; Burg, M.C.; Bunck, A.C.; Heindel, W.; Seifarth, H.; Maintz, D. Dual-source CT angiography of peripheral arterial stents: In vitro evaluation of 22 different stent types. Radiol. Res. Pract. 2011, 2011, doi:10.1155/2011/103873.

29. Valentin, J. Managing patient dose in multi-detector computed tomography (MDCT). ICRP Publication 102. Ann. ICRP 2007, 37, 1-79.

(C) 2015 by the authors; licensee MDPI, Basel, Switzerland. This article is an open access article distributed under the terms and conditions of the Creative Commons Attribution license (http://creativecommons.org/licenses/by/4.0/). 